\title{
METODOLOGIA DE OTIMIZAÇÃO INTEGRADA PARA O DIMENSIONAMENTO DE SISTEMAS DE IRRIGAÇÃO POR GOTEJAMENTO'
}

\author{
Paulo da Costa Medeiros ${ }^{2} \&$ Heber Pimentel Gomes ${ }^{3}$
}

\begin{abstract}
RESUMO
O uso da irrigação por gotejamento vem crescendo nos últimos anos, principalmente pela grande economia no consumo da água que a mesma proporciona, no entanto, por se tratar de um sistema fixo, os elevados custos de instalação tendem a inibir a utilização desta técnica. Buscando-se aproveitar as vantagens que a irrigação localizada oferece, o presente trabalho tem como objetivo fazer uma adaptação no método de otimização econômica integrada (aplicada a sistemas de irrigação por aspersão convencional, desenvolvida por Gomes) à irrigação por gotejamento. Tal metodologia visa à minimização do custo-conjunto da rede de distribuição, energia de bombeamento e equipamentos das parcelas. Os resultados obtidos foram consistentes com a teoria da referida metodologia, o que demonstra sua eficácia, tanto na irrigação por aspersão convencional como, também, no gotejamento.
\end{abstract}

Palavras-chave: sistemas pressurizados, redes de distribuição de água, otimização econômica

\section{INTEGRATED OPTIMIZATION METHODOLOGY FOR DESIGNING TRICKLE IRRIGATION SYSTEMS}

\begin{abstract}
The use of trickle irrigation has increased during the past few years, mainly due to greater economy in water consumption. However, the fact of being a fixed system raises installation costs and inhibits its adoption. In order to get the advantages that localized irrigation offers, the objective of this work is to adopt the integrated economic optimization method (applied to conventional sprinkle irrigation systems, developed by Gomes) to trickle irrigation. This methodology intends to minimize costs of the distribution network, pumping energy and plot equipment. The results obtained were consistent with the methodology used in this work, showing its validity for conventional sprinkle irrigation as well as trickle irrigation.
\end{abstract}

Key words: pressurized systems, water distribution networks, economic optimization

${ }^{1}$ Parte da dissertação de Mestrado do primeiro autor

${ }^{2}$ Mestre em Recursos Hídricos pelo Laboratório de Hidráulica, UFPB - Campus II, Campina Grande. Professor da UFS/DEC/ Recursos Hídricos. Fone: (079) 212 6700, (083) 310 1157, Fax: (083) 310 1388. E-mail: pcosta@ufs.br

${ }^{3}$ Professor Dr. Adjunto do Laboratório de Hidráulica, UFPB - Campus II, CP 505, Campina Grande, PB, Fone: (083) 3101157 , Fax: (083) 310 1388. E-mail: heber@ rechid.ufpb.br 


\section{INTRODUÇÃO}

Otimizar consiste em selecionar, dentre um conjunto de possíveis alternativas, uma que seja ótima, de acordo com determinado critério. O objetivo principal dos diversos métodos de otimização econômica de redes de distribuição de água é encontrar o sistema de menor custo, que atenda aos requerimentos hidráulicos de vazão e pressão nos pontos de consumo. Dois aspectos vêm contribuindo para o aperfeiçoamento das técnicas de otimização: o surgimento do computador pessoal de alta velocidade e a aplicação de técnicas numéricas (Medeiros, 1997).

No caso de redes de distribuição de água para irrigação, as condições iniciais a serem definidas são vazão e pressão requeridas nos pontos de consumo, cotas altimétricas, custos energéticos e preços dos tubos. Deseja-se determinar os diâmetros das tubulações e cotas piezométricas dos principais pontos da rede, inclusive a altura manométrica de bombeamento, de maneira que seja mínimo o custo de investimento e a operação de tal sistema.

Existe uma relação econômica entre a rede de distribuição e a estação de bombeamento. Quando a rede de distribuição é abastecida mediante estação de bombeamento, o dimensionamento das tubulações depende da cota piezométrica de cabeceira. Mantendo-se constante as classes dos tubos disponíveis, quanto maior for esta cota piezométrica, menor será o custo da rede, já que neste caso poderão ser selecionados tubos de menores diâmetros, apesar de que os custos de energia elétrica necessária serão maiores. De maneira oposta, com a diminuição da altura de bombeamento haverá diminuição do consumo de energia e aumento no custo de investimento da rede (utilização de tubulações com diâmetros de maior calibre). Portanto, deve-se encontrar a cota piezométrica ótima da cabeceira da rede, de forma a minimizar a soma dos custos de investimento e de operação do sistema.

Nas duas últimas décadas foram desenvolvidos vários métodos de otimização de sistemas de abastecimento d'água, que proporcionam o custo mínimo de redes de tubulações ramificadas pressurizadas, com seu sistema de bombeamento. Existem várias metodologias de otimização econômica de sistemas de irrigação pressurizada, que envolvem uma quantidade significativa de variáveis admitidas pelos seus modelos de cálculo, em que umas otimizam apenas a parcela de irrigação por gotejamento, como a metodologia proposta por Guimarães Jr. (1993), o qual busca o custo ótimo em função do "layout" da parcela. Outras otimizam através da relação econômica existente entre a altura de bombeamento (custo de energia) e as tubulações da rede coletiva de distribuição (custo das instalações) onde deverá haver uma solução que proporcione o custo mínimo do conjunto: rede de distribuição bombeamento. Exemplo dessas metodologias: a programação linear (fundamentada na relação linear que existe entre o comprimento da tubulação e seu custo); a programação nãolinear (utilização da relação não-linear do custo do tubo com o seu diâmetro) e o método Granados - fundamentado em programação dinâmica - (Granados, 1990).

Este trabalho tem como objetivo adaptar o método de otimização integrada dos sistemas de irrigação sob pressão desenvolvido por Gomes (1992) para sistemas de irrigação por aspersão convencional, à irrigação por gotejamento.

\section{OTIMIZAÇÃO INTEGRADA EM SISTEMA DE IRRIGAÇÃO POR GOTEJAMENTO}

$\mathrm{Na}$ formulação do método de dimensionamento integrado, as pressões requeridas nos terminais da rede de distribuição não são dados fixos do problema e se constituem, também, em variáveis de decisão a serem determinada no processo de otimização. O método parte, dentre outros fatores, de uma série de valores discretos de pressão, para cada terminal da rede de distribuição, que correspondem às várias possibilidades de projeto das instalações de irrigação para cada parcela. Com a seleção das pressões nominais admissíveis dos gotejadores de cada parcela, determinam-se as pressões admissíveis para cada terminal da rede de distribuição. Para tanto, devem ser consideradas as perdas de carga (por atrito e localizadas) e os desníveis geométricos dentro das parcelas.

A Figura 1 mostra a curva característica da parcela: gráfico formado pela união dos pontos cujas coordenadas correspondem às pressões requeridas na tomada d'água de uma parcela por gotejamento $(\mathrm{H})$ e aos respectivos custos das instalações $(\mathrm{P})$ - tubos e acessórios. Cada vértice do gráfico se refere a determinado projeto da parcela (I), caracterizado pelo seu dimensionamento hidráulico.

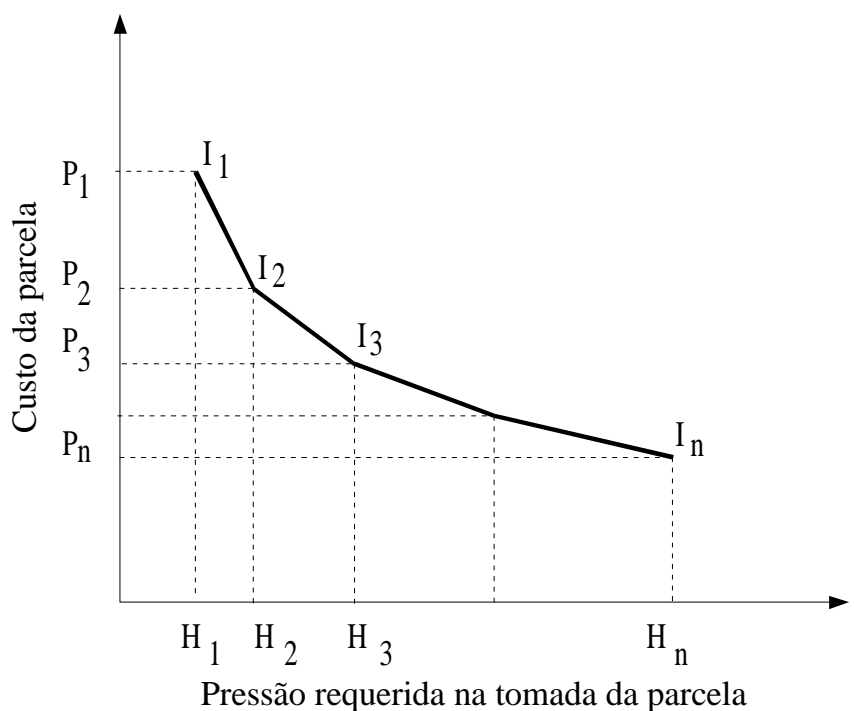

Figura 1. Curva característica da parcela (Gomes,1997)

A declividade (K) de cada segmento da curva característica da parcela é determinada em função das pressões requeridas nas tomadas $\left(\mathrm{H}_{\mathrm{i}}\right)$ e dos preços $\left(\mathrm{P}_{\mathrm{i}}\right)$ das instalações correspondentes; assim, na curva característica da Figura 1, para o segmento que une os vértices correspondentes aos projetos $\mathrm{I}_{2}$ e $\mathrm{I}_{3}$, o gradiente de câmbio $\mathrm{K}_{2,3}$ será igual a:

$$
\mathrm{K}_{2,3}=\frac{\mathrm{P}_{2}-\mathrm{P}_{3}}{\mathrm{H}_{3}-\mathrm{H}_{2}}
$$

em que:

$\mathrm{P}_{2}$ - custo de investimento da parcela correspondente ao projeto $\mathrm{I}_{2}$

$\mathrm{P}_{3}$ - custo de investimento da parcela correspondente ao projeto I3 
$\mathrm{H}_{2}$ - pressão requerida na tomada correspondente ao projeto $\mathrm{I}_{2}$

$\mathrm{H}_{3}$ - pressão requerida na tomada correspondente ao projeto $\mathrm{I}_{3}$

A cada declividade da curva característica da parcela se denomina gradiente de câmbio $(\mathrm{K})$ e representa o custo marginal da diminuição da pressão requerida na tomada da parcela (Gomes, 1992).

\section{PROCESSO OPERATIVO DO MÉTODO DE OTIMIZAÇÃO INTEGRADA}

O processo operativo do método de otimização integrada se baseia no sistema de Granados para otimização de redes e sua metodologia se divide em duas etapas (Gomes, 1997), em que a primeira consiste na determinação de uma solução prévia, que proporciona o custo ótimo da rede de distribuição, mais as instalações das parcelas de irrigação, à cota piezométrica de cabeceira $\left(\mathrm{Z}_{0}\right)$; a segunda etapa compreende uma seqüência de cálculos iterativos na qual, a partir da solução prévia, baixa-se gradualmente a cota piezométrica de cabeceira e se obtém, para cada decréscimo de carga, um novo custo ótimo da rede de distribuição mais as instalações das parcelas de irrigação.

Para a obtenção da solução prévia, se determinam com base no método da velocidade máxima admissível, os diâmetros mínimos admissíveis das tubulações da rede de distribuição e das parcelas de irrigação (que terão as pressões máximas admissíveis em suas tomadas); posteriormente, calculam-se as perdas de carga ao longo da rede e a referente cota piezométrica $\left(\mathrm{Z}_{0}\right)$ estritamente necessária para atender aos requerimentos de pressão nos terminais e superar as perdas de carga de toda a rede.

A segunda etapa do método consiste na execução de um processo iterativo de cálculo, no qual se reduz, passo a passo, a cota piezométrica de cabeceira com o acréscimo mínimo de custo possível da rede de distribuição, mais as instalações das parcelas de irrigação, até alcançar a cota estabelecida pelo projetista.

\section{Aplicação da metodologia}

Para a aplicação da metodologia apresentada neste trabalho, foi utilizada a rede de distribuição esquematizada na Figura 2, a qual mostra 4 parcelas planas, com o mesmo "layout", porém distribuídas em um terreno de relevo heterogêneo, irrigando simultaneamente. Na Figura 3 observa-se o "layout" ou esquema básico das parcelas, e na Tabela 1, são apresentados os dados gerais das mesmas, enquanto na Tabela 2 estão os dados gerais da rede de distribuição, necessários para a aplicação da metodologia anteriormente mencionada.

A Tabela 3, apresenta as velocidades/vazões máximas e os preços para diferentes diâmetros das tubulações e pressões nominais.

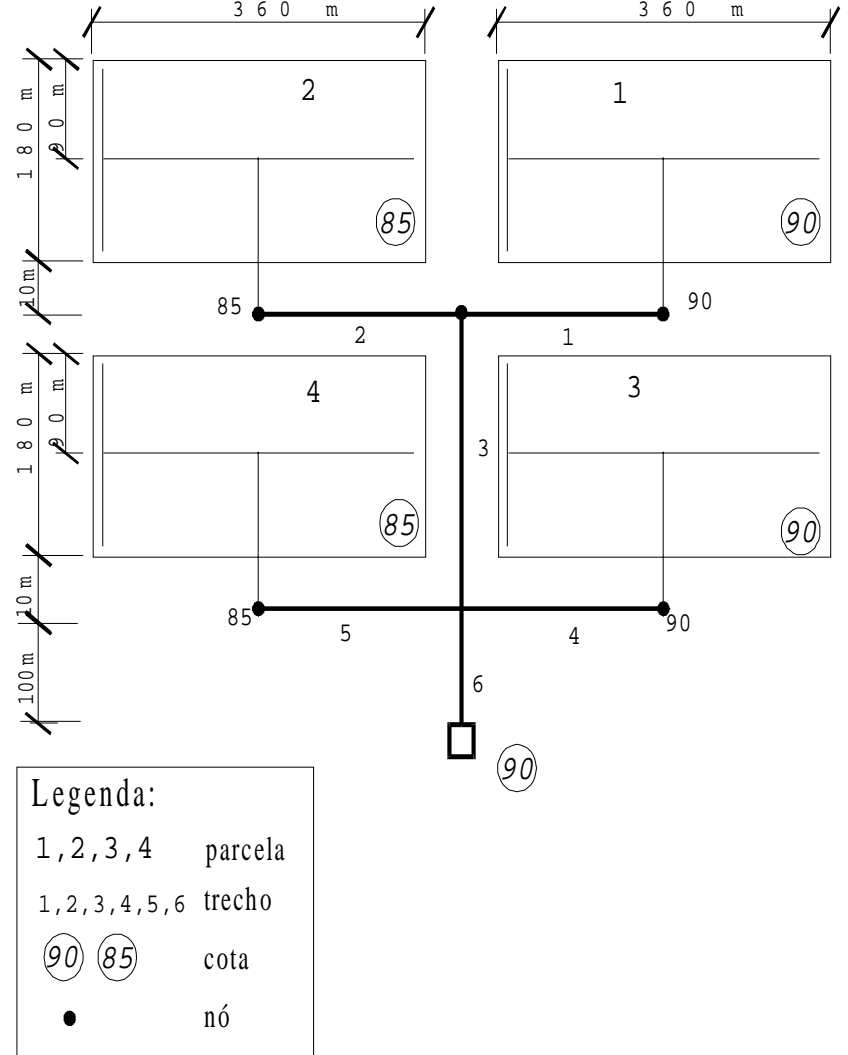

Figura 2. Rede de distribuição referente à aplicação da metodologia de otimização integrada

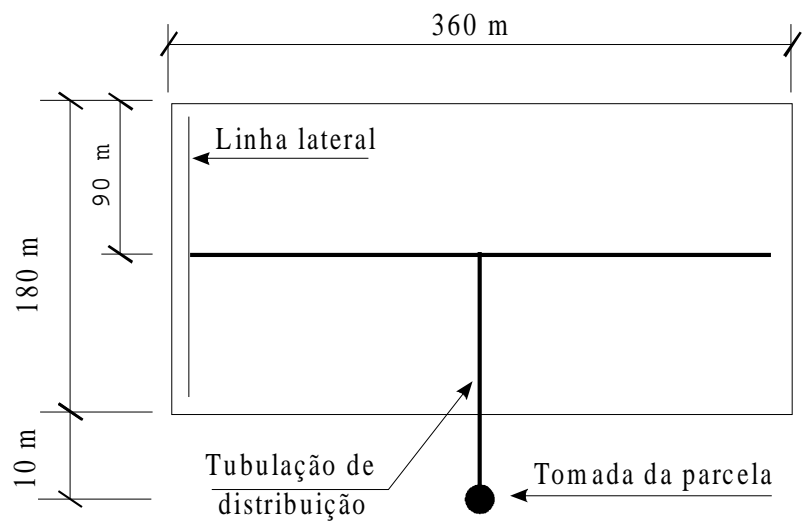

Figura 3. Esquema da parcela de irrigação da rede de distribuição

Tabela 1. Dados gerais da parcela padrão

\begin{tabular}{lr}
\hline Área (ha) & 6,48 \\
Espaçamento entre gotejadores (m) & 1,00 \\
Espaçamento entre linhas laterais (m) & 3,00 \\
Vazão do gotejador $\left(\mathrm{L} \mathrm{h}^{-1}\right)$ & 3,80 \\
Vazão necessária na tomada da parcela $\left(\mathrm{L} \mathrm{s}^{-1}\right)$ & 22,80 \\
Pressão nominal do gotejador (kPa) & 100 \\
Número de laterais & 240 \\
Comprimento de cada linha lateral (m) & 90,0 \\
Número de gotejadores & 21.600 \\
Perda localizada (válv. reg. pressão) na tomada da parcela (kPa) & 50 \\
Número de horas anuais de bombeamento (h) & 2.000 \\
Custo do Kwh (R\$) & 0,05 \\
Coeficiente de atualização de energia & 20 \\
Rendimento esperado do conjunto motor-bomba (\%) & 75 \\
\hline
\end{tabular}

Como os lotes, ou parcelas, apresentam as mesmas dimensões, topografia e "layout", o dimensionamento hidráulico dos mesmos dependerá da pressão disponível nas suas cabeceiras. 
Tabela 2. Dados gerais da rede coletiva de distribuição da Figura 2

$\begin{array}{lr}\text { Área total a ser irrigada (ha) } & 25,92 \\ \text { Número de laterais } & 960 \\ \text { Número de gotejadores } & 86.400 \\ \text { Vazão do trecho 1 }\left(\mathrm{L} \mathrm{s} \mathrm{s}^{-1}\right) & 22,80 \\ \text { Vazão do trecho } 2\left(\mathrm{~L} \mathrm{~s}^{-1}\right) & 22,80 \\ \text { Vazão do trecho 3 }\left(\mathrm{L} \mathrm{s}^{-1}\right) & 45,60 \\ \text { Vazão do trecho } 4\left(\mathrm{~L} \mathrm{~s}^{-1}\right) & 22,80 \\ \text { Vazão do trecho 5 }\left(\mathrm{L} \mathrm{s}^{-1}\right) & 22,80 \\ \text { Vazão do trecho 6 }\left(\mathrm{L} \mathrm{s}^{-1}\right) & 91,20\end{array}$

Tabela 3. Velocidade/vazões máximas ${ }^{1}$ e os preços para diferentes diamêtros $^{2}$

\begin{tabular}{|c|c|c|c|c|c|}
\hline $\begin{array}{l}\text { Diâmetro } \\
\text { Nominal }\end{array}$ & $\begin{array}{c}\text { Pressão } \\
\text { Nominal }\end{array}$ & $\begin{array}{c}\text { Diâmetro } \\
\text { Interno } \\
\mathrm{mm}\end{array}$ & $\begin{array}{c}\text { Velocidade } \\
\text { Máxima } \\
\mathrm{m} \mathrm{s}^{-1}\end{array}$ & $\begin{array}{c}\text { Vazão } \\
\text { Máxima } \\
\mathrm{L} \mathrm{s}^{-1}\end{array}$ & $\begin{array}{c}\text { Preço } \\
\text { por Metro } \\
\text { R\$ }\end{array}$ \\
\hline 12 & 40 & 9,60 & 2,00 & 0,14 & 0,22 \\
\hline 16 & 40 & 13,40 & 2,00 & 0,28 & 0,28 \\
\hline 20 & 40 & 17,00 & 2,00 & 0,45 & 0,40 \\
\hline 25 & 40 & 21,00 & 2,00 & 0,69 & 0,59 \\
\hline 32 & 40 & 27,00 & 2,00 & 1,15 & 0,92 \\
\hline 35 & 40 & 35,70 & 2,00 & 2,00 & 1,96 \\
\hline 50 & 40 & 48,10 & 2,00 & 3,63 & 2,59 \\
\hline 50 & 80 & 46,70 & 2,00 & 3,43 & 3,55 \\
\hline 75 & 40 & 72,50 & 2,00 & 8,26 & 4,79 \\
\hline 75 & 80 & 70,50 & 2,00 & 7,81 & 7,11 \\
\hline 100 & 40 & 97,60 & 2,00 & 14,96 & 8,60 \\
\hline 100 & 60 & 112,60 & 2,00 & 19,92 & 13,58 \\
\hline 100 & 80 & 94,40 & 2,00 & 14,00 & 13,62 \\
\hline 100 & 125 & 108,40 & 2,00 & 18,46 & 24,30 \\
\hline 125 & 40 & 120,00 & 2,00 & 22,62 & 13,11 \\
\hline 150 & 40 & 144,00 & 2,00 & 32,57 & 18,88 \\
\hline 150 & 60 & 162,20 & 2,00 & 41,33 & 24,91 \\
\hline 150 & 125 & 156,40 & 2,00 & 38,42 & 42,22 \\
\hline 200 & 60 & 212,00 & 2,00 & 70,60 & 42,32 \\
\hline 200 & 125 & 204,20 & 2,00 & 65,50 & 70,57 \\
\hline 250 & 60 & 261,60 & 2,00 & 107,50 & 63,07 \\
\hline 250 & 125 & 252,00 & 2,00 & 99,75 & 105,20 \\
\hline 300 & 125 & 299,80 & 2,10 & 148,24 & 149,43 \\
\hline
\end{tabular}

Com os dados das Tabelas 1 e 3, e com base na metodologia de Granados, foram dimensionadas as parcelas, em função da pressão requerida na tomada d'água, buscando-se soluções que proporcionassem custos otimizados das mesmas. Para o cálculo das perdas de carga unitárias ao longo da tubulação, foi utilizada a equação de Hazen-Williams. Os resultados foram acrescidos em 10\%, para levar em conta as perdas localizadas. Após a determinação dos custos parcelares foram obtidos os gradientes de câmbio K (Tabela 4).

Tabela 4. Gradientes de câmbio parcelares - K

\begin{tabular}{ccc}
\hline Pressão na Cabeceira da Parcela - H & $\begin{array}{c}\text { Custo da Parcela } \\
\mathrm{kPa}\end{array}$ & $\begin{array}{c}\text { Gradiente K } \\
\mathrm{R} \$\end{array}$ \\
\hline 200,00 & $10.511,80$ & - \\
150,00 & $11.462,20$ & 190,08 \\
130,00 & $13.823,80$ & $1.180,80$ \\
120,00 & $26.926,90$ & $13.103,10$ \\
\hline
\end{tabular}

\section{RESULTADOS E DISCUSSÃO}

De acordo com a metodologia já citada determinou-se, inicialmente, a solução prévia com base no método da velocidade máxima admissível (Tabela 3).

Após a determinação dos diâmetros mínimos admissíveis para as tubulações dos trechos da rede de distribuição e a obtenção das pressões requeridas máximas nas tomadas das parcelas (terminais da rede de distribuição), calcularam-se as perdas de carga ao longo da rede (utilizou-se a equação de Hazen-Williams) e a cota piezométrica $\left(\mathrm{Z}_{0}\right)$, estritamente necessária para atender aos requerimentos de pressão nos terminais e superar as perdas de carga de toda a rede.

As iterações de cálculo baixam, passo a passo, a cota piezométrica de cabeceira, até que se atinja o valor ótimo, que relaciona o custo da rede mais as instalações das parcelas e o custo da energia de bombeamento capitalizada, por metro de altura de elevação.

A cota piezométrica de cabeceira da solução prévia $\left(\mathrm{Z}_{0}\right)$ é igual a $118,92 \mathrm{~m}$, com o custo correspondente de $\mathrm{R} \$ 164.695,58$ (Tabela 5).

O processo iterativo, que consiste no rebaixamento da cota de cabeceira da rede com o menor acréscimo de custo possível (Tabela 5), mostra que na cota $112,49 \mathrm{~m}$, o custo conjunto mínimo é igual a $\mathrm{R} \$ 153.612,13$, correspondente à soma entre a rede de distribuição ( $\mathrm{R} \$ 53.621,00)$, à energia de bombeamento ( $\mathrm{R} \$ 53.681,53)$ e equipamentos das parcelas de irrigação ( $\mathrm{R} \$ 46.309,60)$.

A Tabela 5 apresenta o custo de todas as iterações para o sistema de distribuição da Figura 2 e, na Tabela 6, observam-se os projetos parcelares a serem utilizados de acordo com a solução ótima.

Em síntese, o projeto que representa o custo ótimo do sistema integrado na condição de contorno, referente à cota de cabeceira da rede de distribuição em 112,49 m, apresenta os resultados contidos no Apêndice.

Tabela 5. Resumo dos custos obtidos de acordo com a metodologia de otimização integrada, segundo a cota piezométrica de cabeceira da rede

\begin{tabular}{ccccc}
\hline $\begin{array}{c}\text { Cota } \\
(\mathrm{m})\end{array}$ & \multicolumn{4}{c}{ Custo $(\mathrm{R} \$)$} \\
\cline { 2 - 5 } & $\begin{array}{c}\text { Rede de } \\
\text { Distribuição }\end{array}$ & $\begin{array}{c}\text { Equip. } \\
\text { Parcelas }\end{array}$ & $\begin{array}{c}\text { Energia } \\
\text { Bombeamento }\end{array}$ & Total \\
\hline 118,92 & $53.621,00$ & $42.047,20$ & $69.027,38$ & $164.695,58$ \\
117,49 & $53.621,00$ & $42,997,60$ & $65.614,54$ & $162.233,14$ \\
113,92 & $53.621,00$ & $43.948,00$ & $57.094,37$ & $154.663,37$ \\
112,49 & $53.621,00$ & $46.309,60$ & $53.681,53$ & $153.612,13$ \\
111,92 & $53.621,00$ & $50.572,00$ & $52.321,16$ & $156.514,16$ \\
110,86 & $58.730,55$ & $50.572,00$ & $49.791,36$ & $159.093,91$ \\
\hline
\end{tabular}

Tabela 6. Projetos parcelares a serem utilizados de acordo com a solução otimizada (cota da cabeceira da rede 112,49 m)

\begin{tabular}{ccc}
\hline Parcelas & Projeto & $\begin{array}{c}\text { Pressão na Tomada } \\
\text { da Parcela }-\mathrm{H}(\mathrm{kPa})\end{array}$ \\
\hline 1 & 3 & 130 \\
2 & 1 & 200 \\
3 & 2 & 150 \\
4 & 1 & 200 \\
\hline
\end{tabular}


O dimensionamento ótimo dos sistemas coletivos de irrigação por gotejamento, mediante o modelo de otimização conjunta, proposto neste trabalho, tem como principal vantagem conseguir maior economia no custo total do sistema, em comparação com os custos obtidos com dimensionamentos efetuados através de outros métodos de otimização já consagrados, que não consideram os custos das parcelas de irrigação como variáveis do sistema.

A relação entre as pressões requeridas na cabeceira da parcela e seu referido custo de instalação é um fator fundamental no dimensionamento do sistema integrado. O método de otimização integrada dos sistemas de irrigação pressurizados facilita a tomada de decisão, com respeito a este aspecto.

Ante a heterogeneidade do terreno (cotas diferenciadas para cada parcela) o método permite otimizar o sistema de distribuição que alimenta um número ilimitado de parcelas (com várias propostas de dimensionamento) obtendo-se, assim, a otimização integrada das mesmas, em conjunto com a rede de distribuição mais energia de bombeamento do sistema.

\section{CONCLUSÕES}

No dimensionamento das parcelas, as linhas laterais direcionaram os custos de investimento das mesmas, visto que, as linhas de derivação e principal apresentaram o mesmo dimensionamento para as 4 propostas de projeto. Tal fato se deve a expressiva densidade de tubulações laterais nos sistemas fixos.

A metodologia de Granados efetivamente busca a solução ótima no dimensionamento da rede de distribuição e dos projetos parcelares. A análise dos custos dos projetos das parcelas de forma integrada, facilita a tomada de decisão, com respeito a uma melhor economia do sistema, visto que chegou-se a solução que melhor proporcionou o custo conjunto (rede de distribuição, mais energia de bombeamento, mais instalação das parcelas de irrigação).

\section{REFERÊNCIAS BIBLIOGRÁFICAS}

CANDE, Tubos e Conexões. Tabela de Preços - Tubos e conexões de PVC e Polietileno . Janeiro/1996. p. 4 e 7.
GOMES, H. P. Dimensionamento optimo del conjunto red coletiva y privadas del riego por aspersion., Universidad Politecnica de Madrid: Tesis Doctoral, 1992.p. 135.

GOMES, H. P. Engenharia de irrigação: Hidráulica dos sistemas pressurizados, aspersão e gotejamento. 3 ed. Campina Grande: UFPB, 1997.p. 282-291.

GRANADOS, A. Infraestructura de Regadios, Redes Coletivas de riego a Presión. Madrid: Servicio de Publicación de la E. T. S. I. de Caminos de la Universidad Politécnica de Madrid, 1990.

GUIMARÃES Jr., J. A. Dimensionamento econômico de uma parcela de irrigação por gotejamento. Campina Grande: UFPB, 1993. p. 63. Dissertação Mestrado

MEDEIROS, P. C. Otimização integrada em sistemas de irrigação por gotejamento, Campina Grande: UFPB, 1997. 98p. Dissertação Mestrado

Apêndice - Resultado do dimensionamento ótimo integrado do sistema de distribuição da Figura 2

\begin{tabular}{|c|c|c|c|c|}
\hline \multirow[t]{2}{*}{ Discriminação } & \multicolumn{4}{|c|}{ Parcelas } \\
\hline & 1 & 2 & 3 & 4 \\
\hline Cota do terreno (m) & 90 & 85 & 90 & 85 \\
\hline $\begin{array}{l}\text { Pressão requerida na } \\
\text { tomada d'água }(\mathrm{kPa})\end{array}$ & 130 & 200 & 150 & 200 \\
\hline $\begin{array}{l}\text { Linha lateral } \\
\text { Diâmetro menoR (mm) }\end{array}$ & $16 / \mathrm{PN} 40$ & $12 / \mathrm{PN} 40$ & $12 / \mathrm{PN} 40$ & $12 / \mathrm{PN} 40$ \\
\hline Comprimento (m) & 14 & 78 & 12 & 78 \\
\hline Diâmetro maior (mm) & 20/PN40 & $16 / \mathrm{PN} 40$ & $16 / \mathrm{PN} 40$ & $16 / \mathrm{PN} 40$ \\
\hline Comprimento (m) & 76 & 12 & 78 & 12 \\
\hline Perda de carga $(\mathrm{kPa})$ & 8,00 & 66,00 & 0,50 & 66,00 \\
\hline Linha de derivação & & & & \\
\hline Diâmetro (mm) & 100/PN60 & 100/PN60 & 100/PN60 & 100/PN60 \\
\hline Comprimento (m) & 180 & 180 & 180 & 180 \\
\hline Perda de carga $(\mathrm{kPa})$ & 11,00 & 11,00 & 11,00 & 11,00 \\
\hline Linha principal & & & & \\
\hline Diâmetro (mm) & 150/PN60 & 150/PN60 & 150/PN60 & 150/PN60 \\
\hline Comprimento (m) & 100 & 100 & 100 & 100 \\
\hline Perda de carga $(\mathrm{kPa})$ & 11,00 & 22,00 & 8,00 & 22,00 \\
\hline Perda de carga total $(\mathrm{kPa})$ & 30,00 & 100,00 & 50,00 & 100,00 \\
\hline Custo (R\$) & $13.823,80$ & $10.511,80$ & $11.462,20$ & $10.511,80$ \\
\hline
\end{tabular}

Rede de distribuição

Trecho 1: Tubulação com diâmetro de 150 mm (PN 125) e comprimento de $180 \mathrm{~m}$ Trecho 2: Tubulação com diâmetro de $150 \mathrm{~mm}$ (PN 125) e comprimento de $180 \mathrm{~m}$ Trecho 3: Tubulação com diâmetro de $200 \mathrm{~mm}$ (PN 125) e comprimento de $180 \mathrm{~m}$ Trecho 4: Tubulação com diâmetro de $150 \mathrm{~mm}$ (PN 125) e comprimento de $180 \mathrm{~m}$ Trecho 5: Tubulação com diâmetro de $150 \mathrm{~mm}$ (PN 125) e comprimento de $180 \mathrm{~m}$ Trecho 6: Tubulação com diâmetro de 250 mm (PN 125) e comprimento de $100 \mathrm{~m}$ Custo das tubulações da rede de distribuição: $\mathrm{R}$ \$ 53.621,00

Energia de bombeamento

Altura manométrica: $112,49-90=22,49 \mathrm{~m}(220,40 \mathrm{kPa})$

Custo capitalizado da energia $\mathrm{R} \$ 53.681,53$ 